\title{
The unresolved issues with risk stratification and management of patients with coronary artery disease undergoing major vascular surgery
}

\author{
[Questions non résolues concernant la stratification du risque et la prise en charge
} des patients atteints de maladie coronarienne subissant une chirurgie vasculaire majeure]

Marie-Claude Parent MD MSc FRCPC, Stéphane Rinfret MD MSC FRCPC

Purpose: The purpose of this article, with a specific focus on patients undergoing vascular surgery, is to review controversial issues related to mechanisms of perioperative myocardial infarction (MI), coronary artery disease detection, and strategies to reduce perioperative complications. We propose explanations for the many conflicting results that have recently emerged in the literature.

Source documents: We searched MEDLINE and reviewed all relevant manuscripts and scientific statements regarding management of patients undergoing non-cardiac surgery.

Principal findings: Identification and prevention of ischemia in patients undergoing vascular surgery remains controversial. While the identification of preoperative ischemia is a marker of a higher perioperative risk, the value of identifying such ischemia has been questioned. We believe this may be, at least in part, due to our limited understanding of perioperative MI. Appropriate management of patients, based on the results of such testing, is likely the key to improving outcomes, and deserves further investigation. Efforts aimed at reducing the ischemic consequences of severe coronary plaques (by revascularization or $\beta$-blocker therapy) have yielded conflicting results. The use of high doses of preoperative $\beta$-blocker therapy may be harmful. Some studies suggest a promising role for statin therapy. Benefits of acetylsalicylic acid must be weighted against the risk of bleeding.

Conclusion: Many questions remain unanswered about the impact of detecting inducible ischemia, and the role of revascularization or $\beta$-blockers in patients undergoing vascular surgery. A better understanding of the pathophysiology of perioperative
$\mathrm{Ml}$ is critical, in order to identify the best approach to improve cardiac outcomes in these patients.

CAN J ANESTH 2008/55:8/pp 542-556

Objectif : L'objectifde cet article est de rendre compte des questions controversées associées aux mécanismes d'infarctus du myocarde (IM) périopératoire, du dépistage des maladies coronariennes et des stratégies de réduction des complications périopératoires, avec une emphase particulière sur les patients subissant une chirurgie vasculaire. Nous proposons une explication aux nombreux résultats contradictoires qui ont récemment vu le jour dans la littérature.

Documents source : Nous avons effectué des recherches dans la base de données MEDLINE et passé en revue tous les manuscrits pertinents ainsi que les déclarations scientifiques concernant la prise en charge des patients subissant une chirurgie non cardiaque.

Constatations principales: Le dépistage et la prévention de l'ischémie chez les patients subissant une chirurgie vasculaire est encore controversée. Bien que le dépistage d'une ischémie préopératoire soit un marqueur d'un risque périopératoire élevé, l'utilité même de l'identification d'une telle ischémie a été remise en question. Nous pensons que cela pourrait être en partie dû à notre compréhension limitée de l'infarctus du myocarde périopératoire. Une prise en charge adaptée des patients basée sur les résultats de tels tests constituerait probablement la clé de l'amélioration des issues cliniques, et cette piste mérite d'être explorée. Les efforts déployés dans le but de réduire les conséquences ischémiques de

From the Division of Cardiology, Department of Medicine, Centre Hospitalier de l'Université de Montréal (CHUM), Université de Montréal, Montréal, Québec, Canada.

Address correspondence to: Dr. Stéphane Rinfret, Centre Hospitalier de l'Université de Montréal (CHUM), 3850 Saint-Urbain,

Montreal, Quebec H2W 1T7, Canada. Phone: 514-890-8000, ext. 14080; Fax: 514-412-7174; E-mail: s.rinfret@umontreal.ca Accepted for publication November 15, 2007.

Revision accepted May 15, 2008. 
plaques coronariennes graves (par revascularisation ou traitement bêtabloquant) ont donné des résultats contradictoires. L'utilisation de fortes doses de bêtabloquants avant l'opération pourrait avoir des conséquences néfastes. Certaines études suggèrent que le traitement à base de statines pourrait être prometteur. Les bienfaits de l'acide acétylsalicylique doivent être soupesés en tenant compte du risque de saignement.

Conclusion: Plusieurs questions demeurent sans réponse concernant l'impact du dépistage de l'ischémie inductible ainsi que concernant le rôle de la revascularisation ou des bêtabloquants chez les patients subissant une chirurgie vasculaire. Une meilleure compréhension de la physiopathologie de l'infarctus du myocarde périopératoire est cruciale afin de déterminer l'approche optimale à adopter pour améliorer les devenirs cardiaques de ces patients.

\section{Vascular surgery and the risk of cardiac events}

Vascular surgery is associated with a relatively high risk of perioperative cardiac events, with two to four times the rate of myocardial infarction (MI) or death of cardiac origin, compared to other types of non-cardiac surgeries. ${ }^{1,2}$ This high-risk surgery causes considerable stress to the heart. Aortic cross-clamping, declamping hypotension, and the associated fluid shifts are some of the elements of surgical trauma that induce an inflammatory, hypercoagulable and hypoxic state. These events can trigger perioperative ischemia and MI, especially in patients with coronary artery disease (CAD). ${ }^{3,4}$ Coronary artery disease is common in patients undergoing vascular surgery and is the leading, long-term cause of death in patients with peripheral artery disease (PAD) who survive their surgery. ${ }^{5,6}$ Perioperative $\mathrm{MI}$ is associated with increased in-hospital mortality rates ${ }^{1,7}$ and an 18 -fold increase in long-term reinfarction or mortality. ${ }^{8}$ Increased cardiac troponin, which is highly specific for MI, ${ }^{9}$ has been recently identified in up to $24 \%$ of patients after major vascular surgery, and confers an adverse long-term prognosis. ${ }^{10}$

In 2003 , approximately 250,000 peripheral bypass, carotid endarterectomy, and aortic aneurysmectomy procedures were performed in the United States. ${ }^{\mathrm{A}}$ Each year, it is estimated that treatment of cardiac complications following non-cardiac surgery accounts for approximately $\$ 20$ billion of health care expenditures in the United States. ${ }^{11}$ Despite recent advances in the management of cardiac patients undergoing non-cardiac surgery, perioperative cardiac morbidity and mortality remain important concerns among

\footnotetext{
A Trend in Procedures for Peripheral Vascular Disease: 7-Year Nationwide Statistics. Available from URL; http://scvs. vascularweb.org/SCVS_Contribution_Pages/Abstracts/2006/ SCVS06_AbstractMP22.html.
}

health care providers. ${ }^{3,12,13}$ These facts underscore the importance of increasing our understanding of the mechanisms and risk factors of perioperative adverse cardiac events, which should facilitate the improvement of interventions aimed at reducing these risks.

Many issues regarding this surgical population remain controversial and unresolved at the present time, despite years of research aimed at identifying the mechanisms of perioperative MI or death, detecting patients at higher risk for such complications, and selecting the most appropriate management to decrease these adverse events. Therefore, the purpose of this article, with a specific focus on patients undergoing vascular surgery, is to review and discuss the controversies related to the mechanism of perioperative MI, CAD detection, and strategies to reduce perioperative complications (Table).

\section{The uncertain pathophysiology of perioperative myocardial infarction}

In order to identify the best perioperative management strategy, we need to better understand the pathophysiology leading to these events. While plaque rupture and fissure, followed by superimposed thrombosis, have long been identified as the leading cause of spontaneous MI in the non-surgical context, ${ }^{14,15}$ the pathophysiology of perioperative MI is less clear. In the non-surgical setting, angiographic studies have shown that most MIs result from the rupturing of vulnerable plaques, which, as a rule, did not significantly encroach the vessel lumen prior to rupture. ${ }^{16,17}$ In one landmark study, $66 \%$ of MIs occurred on plaques that were less than $50 \%$ in severity. ${ }^{17}$ Often, the segment responsible for the MI is not the one that presented the most severely stenotic plaque on previous coronary angiography. ${ }^{18}$ Vulnerable small plaques (often covered by a thin fibrous cap and filled with a large lipid core and macrophages) are believed to be more prone to rupture than more severe and fibrous plaques. ${ }^{16}$ These small plaques are often difficult to visualize with traditional invasive coronary angiography. ${ }^{16,19}$ In a large study prospectively evaluating non-bypassed coronary segments in patients randomized in the Coronary Artery Surgery Study trial, the risk of spontaneous MI or coronary occlusion was conversely, and somewhat contradictorily, proportional to the baseline severity of the lesion, with severe plaques being at a much higher risk of subsequent occlusion. Only $0.7 \%$ and $2.3 \%$ of segments with narrowing of $<5 \%$ and 5 to $49 \%$, respectively, resulted in coronary occlusion during the five-year follow-up. In contrast, occlusion occurred in close to one-fourth of lesions that were $>80 \%$ in severity on the initial angiography. ${ }^{20}$ 
TABLE Certainties and unresolved issues with risk stratification and management of patients with coronary artery disease undergoing major vascular surgery

\begin{tabular}{lll}
\hline Topic & Certainties & Unresolved issues and areas of future research \\
\hline Perioperative myocardial infarction & $\begin{array}{l}\text { Associated with adverse outcomes during } \\
\text { follow-up }\end{array}$ & $\begin{array}{l}\text { Pathophysiology is uncertain: contribution of } \\
\text { severe plaques vs vulnerable plaques }\end{array}$
\end{tabular}

Higher risk during vascular surgery compared to other non-cardiac surgeries

Stratification based on clinical factors Several risk indices developed, with good clinical performances

Functional testing

Excellent negative predictive value

Incremental predictive value compared to clinical risk assessment alone

Coronary angiography

Can adequately identify high-risk coronary anatomy

Associated with increased risk of complications in peripheral artery disease patients

Revascularization

Beta-blocker therapy

Statin therapy

Acetylsalicylic acid (ASA)
Good clinical outcomes in patients following revascularisation

No incremental benefit compared to medical therapy

Early discontinuation of antiplatelet therapy following coronary stenting is harmful

Good protection against perioperative clinical outcomes in patients with low heart rates

No incremental benefits of metoprolol in several large recent trials

Useful in stable coronary artery disease

Reduces events in stable and unstable coronary artery disease
No clear diagnostic criteria to identify myocardial infarction

Stratification, based on clinical risk factors alone, may be sufficient in intermediate-risk factors

Usefulness of detecting ischemia prior to vascular surgery is questioned

Non-invasive coronary angiography with cardiac tomography could be useful to assess coronary anatomy and could help management prior to vascular surgery

Is medical treatment enough for patients with high-risk coronary anatomy, such as left-main coronary disease?

High doses may be harmful

Duration of therapy is unclear

Class effects of beta-blockers?

Value of statin therapy to reduce perioperative events is unknown

Risk $v s$ benefits unclear in patients undergoing vascular surgery
However, small plaques outnumber, by far, the more severe and significant plaques in a coronary tree. ${ }^{16,18}$ Pathological studies have shown that plaque rupture depends on plaque composition, rather than on plaque size. ${ }^{16,21,22}$ Also, factors associated with increased risk of subsequent rupture, such as a large lipid pool or a thin fibrous cap, ${ }^{16}$ poorly correlate with the severity of the plaque by intravascular ultrasound. ${ }^{23}$ Ruptured plaques are often the same size as non-ruptured adjacent plaques in the same vessel, but are often more eccentric. ${ }^{24}$ In a ruptured plaque, the percent stenosis may be lower than in a non-ruptured plaque, ${ }^{23}$ because of the compensatory vascular enlargement phenomenon or the negative remod- elling of the coronary vessel. ${ }^{24}$ Therefore, smaller plaques might lead to more MIs, simply because they occur more frequently.

The impact of plaque severity on the risk of perioperative MIs remains uncertain. ${ }^{3,4}$ With the exception of total occlusions, which were associated with an increased risk of death or MI, one case-control study showed that complications following vascular surgery correlated more closely with an increased number of lesions $>30 \%$ than with the severity of the lesions on previous coronary angiography. ${ }^{25}$ However, in a study of patients who died as a result of perioperative MI, significant stenoses (> 50\%) were present in the majority of patients (93\%) [with three-vessel 
disease or left main disease (LMD) present in 44\%]. Although plaque rupture or hemorrhage was present in more than half of the patients, they occurred most often on non-significant plaques. ${ }^{26}$ In a series of 26 patients who died as a result of a perioperative MI, plaque rupture was also identified in nearly half of the cases. Fifty four per cent of the patients had threevessel disease. ${ }^{27}$ However, perioperative MIs are often preceded by sustained, stress-induced ST depressiontype ischemia. ${ }^{28}$ Such an observation implies that severe stenoses have an important impact on the risk of adverse events. ${ }^{4}$ It is well known that non-perioperative MI can result from sustained ischemia distal to severe coronary stenoses in the setting of anemia, fever, or other factors that increase oxygen demand. Therefore, some perioperative events may be attributable to the same factors. Moreover, absence of inducible ischemia on a preoperative test, which suggests the lack of severe stenoses, is also associated with an extremely high negative predictive value for perioperative MI. ${ }^{12}$ In summary, the pathophysiology of fatal and non-fatal perioperative MI, especially with respect to the relative contribution of severe ps vulnerable plaques, deserves further evaluation. Based on available evidence, it appears that perioperative MI may result from rupture of vulnerable plaques, but also, to a certain extent, as a result of sustained ischemia distal to severe stenoses. The relative contribution of each mechanism is not well understood, and speaks to the difficulty of evaluating the risk of adverse cardiac events in patients undergoing non-cardiac surgery. Further studies are needed to determine whether, and in what manner, perioperative infarction differs from non-operative infarction, and how this pathophysiology might influence preoperative evaluation and perioperative management.

\section{Definition and monitoring of perioperative myo- cardial infarction}

Although relatively infrequent, perioperative MI remains a serious complication after non-cardiac operations. Recent data from the Patient Safety in Surgery Study ${ }^{29}$ reported postoperative cardiac adverse events (death, MI, or cardiac arrest) in 2,362 patients out of 183,069 patients (1.29\%) following general or vascular surgery. However, in this study, authors used a fairly stringent definition of acute MI, only being considered when transmural or new $\mathrm{Q}$ waves are present on an electrocardiogram (ECG). Thoracoabdominal vascular surgery and peripheral vascular surgery were associated with the highest rates of cardiac events (3.2\% and $2.3 \%$, respectively). More than half (59\%) of patients who experienced an adverse event died within 30 days of surgery. These results did not take into account the fact that the majority of perioperative MIs are silent and without ST segment elevation, ${ }^{29}$ and, therefore, they may represent an underestimate of the clinical impact of perioperative MI. In a study of patients undergoing vascular surgery, all infarctions were non-Q-wave, and were detected by a rise in cardiac troponin-I (observed more than $20 \%$ of patients) during, or immediately after, a prolonged, ST depression-type ischemia. In the context of a major vascular surgery, postoperative CK-MB and troponin elevations, even at low cut-off levels, are independent and complementary predictors of long-term mortality. ${ }^{10}$

The definition of a perioperative MI will certainly have an impact on its incidence. Although there are no standardized diagnostic criteria, cardiac troponins and ECG now play an important role in the diagnosis of this complication. The ECG obtained immediately after surgery allows a reassessment of the patient's risk. The occurrence of new ischemic changes on ECG will have an unfavourable impact on the subsequent outcome, and these patients may benefit from closer follow-up. ${ }^{30}$ The following factors should be considered, in addition to a typical increase in troponin levels, or a typical fall after an elevated value, without an alternative explanation (e.g., pulmonary embolism): $:^{9,13,31}$

- Signs or symptoms of ischemia (including dyspnea);

- Appearance of new Q waves on the ECG, or changes indicative of ischemia;

- New (or presumed new) regional wall motion abnormalities on echocardiography or perfusion defects, as visualized with nuclear medicine imaging;

- Presence of new Q waves on the ECG, in a context where troponins were not measured (constitutes a reliable diagnostic criterion of perioperative $\mathrm{MI}$ ).

According to the ACC and the European Society of Cardiology, MI is defined by an increase in levels of troponins $\mathrm{T}$ or I beyond "a value exceeding the $99^{\text {th }}$ percentile of a reference control group", at least once during the first $24 \mathrm{hr}$ following the index event. ${ }^{9}$ Although several studies have shown that an elevated troponin is associated with poorer short- and longterm prognosis, ${ }^{32,33}$ the use of troponins as a single indicator of perioperative MI remains controversial. Indeed, some studies have not revealed an association between postoperative troponin rise and an adverse impact. ${ }^{34}$ This is emphasized in the most recent ACC/ AHA guidelines published in 2007; routine measurement of cardiac troponins after surgery cannot be recommended, but should be performed in the presence of ECG changes or typical chest pain. ${ }^{35}$ 
Preoperative assessment of the risk of cardiac events; clinical risk factors and inducible ischemia Clinical assessment of perioperative risk

In general, risk evaluation is based on the use of clinical markers, the patient's functional capacity, and the specific risks related to surgery. ${ }^{35}$ First, in the process of evaluating the severity and/or stability of the condition, a complete medical history must be done. It is well known that the risk of perioperative cardiac events is particularly high in patients who have suffered an MI within the six months preceding vascular surgery. ${ }^{12,36,37}$ A prospective study demonstrated that the more recent the occurrence of MI, the higher the risk of reinfarction in a surgical context. ${ }^{38}$ Patients with unstable angina or acute heart failure are also at a very high risk for cardiac complications. ${ }^{12}$ Consequently, after an MI, it is recommended to wait a minimum of four to six weeks before elective surgery. ${ }^{35}$ It is also essential to carry out a targeted physical examination, in order to diagnose any significant cardiac pathology. Aortic stenosis and decompensated heart failure are two critical entities to diagnose/exclude, as they are associated with a high risk of cardiac complications.

Perioperative evaluation becomes more challenging when stratifying risk from clinical predictors of intermediate risk. ${ }^{12}$ Numerous factors have been identified as being associated, independently or not, with perioperative coronary ischemic events. ${ }^{12}$ In patients undergoing vascular surgery, Eagle et al. ${ }^{36}$ identified five independent factors of perioperative complications; presence of $\mathrm{Q}$ waves, history of angina, treatment of ventricular ectopies, diabetes, and age $>70$ yr. Lee et $a l^{2}{ }^{2}$ published the Revised Cardiac Risk Index (RCRI), the most contemporary scoring system derived from a cohort of 2,898 patients undergoing non-cardiac surgery (21\% vascular). Six independent predictors were identified: high-risk surgery, ischemic heart disease, congestive heart failure, cerebrovascular disease, perioperative treatment with insulin, and preoperative serum creatinine $>175 \mu \mathrm{mol} \cdot \mathrm{L}^{-1}$. The RCRI class can be calculated for the presence of factors as follows: class I, for no factors; class II, for one factor; class III, for two factors; and class IV, for three or more factors. In a validation cohort of 1,422 patients, the index performance was excellent, with a c-statistic for the whole validation cohort of 0.81 , suggesting excellent predictive value for the combined incidence of major cardiac complications. However, while the performance of the index remains very good in patients undergoing vascular surgery (c-statistic of 0.77 ), the index performed poorly with respect to patients undergoing abdominal aortic aneurysm surgery (c-statistic of 0.54$).{ }^{2}$ Due to the lower predictive ability of clinical indices in vascular surgery patients, coupled with earlier evidence of the value of perioperative revascularisation or $\beta$-blockers to reduce cardiac risk (from retrospective studies or small randomized trials), the ACC/AHA task force used to recommend testing to detect inducible ischemia in moderate-risk patients undergoing high risk surgery such as vascular surgery. ${ }^{12}$ However, in light of the new studies available that cast some doubts on the value of functional stratification and prophylactic revascularization before surgery, they recently revised their recommendations, simplifying the approach to risk assessment. ${ }^{39,40}$ They also integrated the Lee index ${ }^{2}$ as criteria to assess for the further need for non-invasive functional assessment. According to these recommendations, patients who do not present any of the risk factors, according to the RCRI, can proceed to surgery without additional investigation. In the presence of one of these factors (history of $\mathrm{CAD}$, heart failure or stroke, diabetes treated with insulin, or renal failure), it is recommended that a functional investigation be considered, but only if it is likely to modify the patient's management. ${ }^{35}$

\section{Functional assessment aimed at detecting inducible ischemia}

The presence of inducible coronary ischemia, in up to $40 \%$ of patients, is clearly associated with increased risk. ${ }^{12}$ Because of poor circulation of the lower limbs, patients with PAD, have a significant exercise limitation that often makes the stress test impossible for practical cardiac evaluation. It has been estimated that 30 to $60 \%$ of patients referred for perioperative stratification cannot adequately undergo the treadmill stress test. ${ }^{36,41}$ As a result, pharmacological testing [using dipyridamole myocardial perfusion scintigraphy (MPS) or dobutamine echography (DE)] is often used in the preoperative context. Myocardial perfusion scintigraphy has been used for over 20 years to detect $\mathrm{CAD}$ and to assess prognosis. It involves the injection of a radioactive tracer which is absorbed by viable myocardial cells where flow is preserved. Images that result from the absorption of the tracer reflect both viability and perfusion. When compared to resting images, stress imaging (pharmacologic or effort) indicates areas of reduced perfusion (reversible defects) or infarction (fixed defects). ${ }^{42}$ When using DE, the presence of a new segmental contractile abnormality during infusion also indicates ischemia. In one recent meta-analysis comparing the performance of six stratification tests often used to predict events, MPS and DE had comparable sensitivities (83\% and $85 \%$, respectively), but the specificity of DE was higher 
(70\%) than with MPS (49\%). ${ }^{43}$ In general, with both tests, it is recognized that the absence of inducible ischemia prior to the vascular surgery, is a marker of a very good prognosis, with a negative predictive value ranging from 97 to $100 \%$ in the majority of studies. ${ }^{12}$

Boersma et al. ${ }^{44}$ assessed the incremental value of preoperative testing with DE over clinical risk assessment with the RCRI for the prediction of perioperative events in 1,351 patients undergoing vascular surgery. Using multivariate modelling, the study confirmed that prior MI, angina, heart failure, and cerebrovascular disease (all factors of the RCRI) were independent clinical risk factors for complications; but it also identified age $>70$ to be independently associated with an increased risk in that selected population. Preoperative inducible ischemia and $\beta$-blocker therapy had additive predictive value over the RCRI alone. Therefore, for patients being considered for vascular surgery, the use of functional testing improves risk stratification, even when controlling for the RCRI class.

However, the positive predictive value (i.e., the probability of a perioperative event in the presence of ischemia) was less than 20 to $25 \%$ in most series. ${ }^{12}$ Therefore, more than three- quarters of patients with preoperative inducible ischemia will not experience perioperative MI or death. Rather than considering test results as "positive" or "negative", the extent of ischemia - measured as the proportion of the myocardium that shows reversible perfusion defects or contractile abnormalities - can improve the positive predictive value of the functional test. A meta-analysis of all studies that assessed MPS prior to vascular surgery showed that the presence of ischemia, in $20 \%$ or fewer segments, did not significantly increase the risk of complications. However, greater areas of reversibility on MPS were associated with increased risk. ${ }^{45}$ Recently, in the second Dutch Echocardiographic Cardiac Risk Evaluation Applying Stress Echo (DECREASE-2) study ${ }^{40}$ the extent of ischemia on the functional test was also highly predictive of events. Intermediaterisk patients, who were managed with $\beta$-blockers and had no preoperative ischemia on DE or MPS, had no events. However, low to moderate ischemia ( $1-4$ ischemic segments) was associated with an OR of 42 , while extensive ischemia ( $\geq 5$ ischemic segments) was associated with a very high OR of $107 . .^{40}$

Therefore, the presence of preoperative inducible ischemia is a strong predictor of cardiac complications after vascular surgery. However, surprisingly, recent data suggest that identifying such preoperative inducible ischemia in moderate-risk patients is not associated with improved outcomes during and after the surgery. In the second Dutch Echocardiographic Cardiac Risk
Evaluation Applying Stress Echo (DECREASE-2) study, ${ }^{40} 770$ intermediate-risk patients were randomized to two groups: the first group was tested by MPS or $\mathrm{DE}$, in order to detect inducible ischemia; the second group was not tested at all. All patients were managed with $\beta$-blockers, with a target heart rate of less than 65 beats. $\mathrm{min}^{-1}$. They found no benefit in testing, as there were similar rates of perioperative death or MI ( $2.3 \%$ with testing $v s$ s $1.8 \%$ without testing) in these patients with tight heart rate control. Somewhat in contrast to these findings, as mentioned earlier, the study also showed that the extent of ischemia on the functional test was highly predictive of events - and this is consistent with other studies. ${ }^{12}$

Given that ischemia is a strong predictor of events, how can we explain the fact that identifying the presence of such ischemia prior to the surgery was not associated with improved outcomes in DECREASE2 ? Does this imply that functional stratification prior to vascular surgery has become obsolete? The answer lies within the meaning of such ischemia, and the therapeutic interventions which unfold when such ischemia is identified. First, it is tempting to conclude that severe coronary plaques leading to perioperative ischemia are the likely cause of perioperative events. Rather, such "positive" tests may be markers of a larger, more extensive coronary artery atherosclerotic burden, with an increased number of plaques, some of which may be vulnerable. ${ }^{4}$ However, the current medical and surgical management of patients undergoing vascular surgery may not always be appropriately targeted to reduce the risk of plaque rupture. Nevertheless, numerous studies, including DECREASE-2 trials, have demonstrated that the extent of inducible ischemia is predictive of cardiac events in the perioperative period. ${ }^{12}$ At a minimum, these factors can inform clinicians of the safety of pursuing the planned surgery. ${ }^{3}$ Reduced long-term mortality in patients who had previously undergone preoperative testing (with or without coronary revascularization) has been seen in observational studies. ${ }^{6}$ In DECREASE-2, only moderate-risk patients, with one or two clinical risk factors and several ischemic segments, were considered for coronary angiography. It is not known how patients with $\geq$ three risk factors were managed. After randomization, only 12 patients with extensive ischemia underwent revascularization. Patients with less extensive ischemia were not considered for coronary angiography. Therefore, with a limited number of patients who underwent revascularization in the testing arm, and with a low rate of coronary angiography after a positive test, it is not surprising that they did not observe any difference in clinical outcomes 
compared with the non-testing group. It is unknown if a more aggressive management of patients with ischemia (lower criteria for coronary angiography) would have allowed for increased detection of highrisk anatomy such as LMD. Patients with significant LMD are expected to derive a large short- and longterm benefit from coronary artery bypass graft surgery $(\mathrm{CABG}){ }^{46}$ The extent of ischemia on the functional test was also a predictor of perioperative events in the DECREASE-2 trial, which suggests that patients with high-risk coronary anatomy might have been inappropriately managed as a consequence of the testing. Finally, the DECREASE- 2 trial used a relatively stringent definition of MI. Post-MI was only considered in the presence of both a $\mathrm{Q}$ wave and a rise in cardiac enzymes. However, it is well known that the majority of perioperative MI episodes are without ST elevation or a $\mathrm{Q}$ wave. ${ }^{28}$ For these reasons, it would be premature to conclude that risk stratification, beyond the simple count of clinical risk factors, is no longer required in patients undergoing vascular surgery.

Are we testing with the appropriate methods? In the perioperative setting, we propose that the best test should have a high specificity and sensitivity for the detection of very high risk anatomy (such as significant LMD), for which the management would definitely be altered as a result of the tests. Indeed, LMD is clearly associated with increased long-term adverse prognosis and should justify revascularization with $\mathrm{CABG}$ prior to the vascular surgery, irrespective of the forthcoming non-cardiac surgery. Although no study has ever proved the impact of revascularization of LMD prior to non-cardiac surgery, CABG is a class 1 recommendation for such an anatomy. ${ }^{46}$ Unfortunately, the ability to detect adverse coronary anatomy, such as LMD, is limited, especially with MPS. It is known that the diagnostic accuracy of dipyridamole MPS for the detection of significant coronary stenoses, confirmed with a coronary angiography, is usually excellent. Sensitivity ranges between 90 and $97 \%$, and specificity from 28 to $100 \%$, with most around $70 \%{ }^{42}$ However, in studies that assessed the diagnostic accuracy of MPS, all patients underwent a coronary angiography (the gold standard). While more extensive ischemia has been associated with increased perioperative risk, ${ }^{3,12}$ the accuracy of MPS in detecting LMD continues to be debated. High-risk perfusion patterns suggestive of LMD have been proposed, though with low sensitivity ranging from 13 to $29 \% .{ }^{47-50}$ In a series of 43 patients with documented significant LMD, Nygaard et al. ${ }^{48}$ found that only $67 \%$ demonstrated high-risk scintigraphic findings suggestive of LMD. While a LMD pattern (an extensive homogeneous defect over the whole anterolateral segment and simultaneous defects in the high anteroseptal and high posterolateral segment) was $100 \%$ sensitive for very severe (>90\%) stenoses, Wakasugi et al..$^{51}$ found that this pattern performed poorly in identifying LMD of lesser severity. In the largest series of MPS involving isolated LMD, no unique patterns of ischemia were found. Four of 25 patients had normal MPS. ${ }^{52}$ In summary, detecting inducible ischemia, especially with MPS, may not be the most effective technique to detect high-risk anatomy, unless patients with inducible ischemia are systematically referred for coronary angiography, irrespective of the extent of ischemia. Also, there are no published data evaluating the sensitivity and specificity of DE for the identification of significant LMD. One study showed that dipyridamole echocardiography has a high sensitivity for LMCA disease, although the ability or specificity to distinguish significant LMD from three-vessel disease was low. ${ }^{53}$ Again, unless coronary angiography is performed to detect LMD resulting from a positive $\mathrm{DE}$, such anatomy might be overlooked. One study reported two cases of significant LMD that failed to be identified with DE prior to non-cardiac surgery. Both patients experienced a major perioperative event. ${ }^{54}$ Therefore, studies on alternative methods for the stratification of patients undergoing non-cardiac surgery are needed.

\section{Diagnostic coronary angiography}

As stated in the most recent ACC/AHA guidelines, indications for an invasive preoperative coronary angiography are similar to those described for the nonoperative setting. There is no clear recommendation documenting a threshold of ischemia that would justify a coronary angiography. Therefore, it is the clinician's responsibility to take patients' specific factors into account before referring the patients for an invasive, coronary angiography. In fact, there are potential downsides associated with a coronary angiography, since many patients with coronary stenosis suitable for revascularization are likely to undergo percutaneous coronary intervention (PCI) or CABG. Opinions differ among cardiologists regarding the role of prophylactic coronary revascularization for patients scheduled for vascular operations, with cardiologists often deviating from published guidelines as to their use of medical therapy, PCI, and CABG. ${ }^{55}$ Liberal use of coronary revascularization may bear important health consequences, especially if stenting is performed.

After ruling out adverse coronary anatomy, interventional cardiologists should be encouraged not to proceed with coronary revascularization without consultation with the referring physician, if such a 
revascularization is not expected to change the perioperative and long-term prognosis of the patient. Indeed, one recent large randomized trial, involving coronary stenting in stable coronary patients, failed to demonstrate protection from future cardiac events. ${ }^{56}$ Most patients managed in the perioperative setting will have stable coronary disease, which can be properly controlled with medical therapy.

On the other hand, imaging of the coronary tree may have a significant prognostic value, in the context that perioperative mortality from vascular surgery can reach up to $14 \%$ in patients with severe, non-bypassed or inoperable CAD. ${ }^{57}$ Therefore, it would be premature to conclude that coronary angiography is no longer required, even if coronary revascularization were not shown to be beneficial in recent studies.

Therefore, to prevent cardiologists from performing prophylactic preoperative PCI in patients planned for major non-cardiac surgery, the best approach might be to avoid the catheterization laboratory. Non-invasive, coronary angiography with multi-detector cardiac computed tomography (MDCT), especially with the latest generation of 64-slice detectors, is a novel, but mature, imaging modality that has been consistently shown to be effective in the detection of CAD in large coronary vessels. The positive predictive value of a suspected stenosis on the left main trunk is over $95 \%$, whereas its negative predictive value approaches $100 \% .{ }^{58}$ Multi-detector cardiac computed tomography angiography has never been studied in the setting of perioperative evaluation. Its capacity to assess coronary anatomy and cardiac risk prior to vascular surgery appears promising, but needs further investigation.

\section{Preoperative coronary revascularization with CABG or PCI}

Although revascularization with $\mathrm{CABG}$ or angioplasty without stenting, prior to non-cardiac surgery, has been associated with extremely low rates of ischemic complications in observational studies, ${ }^{57,59,60}$ the role of preoperative revascularization, to protect CAD patients undergoing vascular surgery, had not been evaluated until recently. Two trials assessed the value of prophylactic revascularization prior to vascular surgery. First, the Coronary Artery Revascularization Prophylaxis (CARP) trial $^{39}$ assessed the incremental value of revascularization (PCI or CABG) over medical therapy prior to major vascular surgery. This study randomized 510 patients [with at least one significant $(\geq 70 \%)$ stenosis in a major coronary artery] to revascularization (PCI or CABG) vs no revascularization. Three-vessel disease was present in one-third of patients. Patients with non-obstructed coronary arteries, patients with
CAD considered non-amenable to revascularization, or patients with LMD were excluded. In this population, this trial failed to demonstrate any benefits with revascularization, as there were identical rates of perioperative MI (8.6\% in both groups) and similar in-hospital and long-term mortality $(22 \%$ with revascularization $v s$ $23 \%$ without revascularization, at a median follow-up of $2.7 \mathrm{yr}$ ). Revascularization delayed the vascular surgery by one month. Therefore, in absence of high-risk clinical factors such as unstable angina or a recent $\mathrm{MI}$ mandating a coronary revascularization, CAD patients undergoing major vascular surgery do not appear to benefit from alleviating the effects of severe coronary stenosis with stenting or CABG. This study, however, was limited by the relatively small sample size and by the number of high-risk patients that were screened, but eventually excluded, from randomization. Nevertheless, the CARP trial strongly questions the impact of preoperative revascularization on early postoperative outcome and on long-term mortality.

Although this trial failed to demonstrate the benefits of revascularization in patients with CAD, it is important to emphasize that it did not test the value of a diagnostic coronary angiography to further assess the risk. In fact, in order to recruit patients, investigators of the CARP trial were encouraged to refer all suspected high-risk patients for a coronary angiography. A functional test was not required to refer patients for a coronary angiography and was performed on only $62 \%$ of patients. As a result, of the 1,190 patients who underwent coronary angiography, only 510 (43\%) were deemed amenable to revascularization and randomized in the study. Of the 680 patients excluded from randomization, 363 (31\% of all coronary angiographies) were excluded because of non-obstructive CAD, 215 (18\%) because the CAD was considered non-amenable to revascularization, and $54(4.5 \%)$ because of significant LMD. ${ }^{39}$ Patients with significant left ventricular dysfunction were also excluded.

The fifth Dutch Echocardiographic Cardiac Risk Evaluation Applying Stress Echo (DECREASE-V) study is the second trial that addressed the value of revascularization prior to vascular surgery. This study was designed to recruit high-risk patients, i.e., those with three risk factors (over $70 \mathrm{yr}$ of age, angina pectoris, prior MI, heart failure, diabetes, renal dysfunction, or prior stroke or transient ischemic attack) with extensive stress-induced ischemia using DE or stress nuclear imaging ( $\geq 5 / 17$ ischemic segments or $\geq 3 / 6$ ischemic walls). Perioperative $\beta$-blocker therapy was used for all patients, before randomization to either an invasive approach, followed by revascularization (PCI or $\mathrm{CABG}$ ), or to a non-invasive, medical-only strat- 
egy. The $\beta$-blocker dose was adjusted in all patients to achieve a resting heart rate of 60 to 65 beats $\mathrm{min}^{-1}$. A total of 1,880 vascular surgery patients were screened, but only 101 patients were included in the pilot trial. Of these, 49 patients were randomized to coronary revascularization, and 51 patients were assigned to medical treatment. It is worth noting that a reduced left ventricular ejection fraction $(<35 \%)$ was observed in 43 patients $(43 \%)$. The primary endpoint was the composite of all-cause death and non-fatal MI. All patients randomized to the invasive strategy had a coronary angiography revealing; a two-vessel disease in 12 patients $(24 \%)$, a three-vessel disease in 33 patients $(67 \%)$, and a LMD in four patients $(8 \%)$. Complete revascularization was achieved in 42 patients (86\%). The incidence of all-cause mortality or non-fatal MI was $43 \%$ in the preoperative revascularization group vs $33 \%$ in the medical treatment only group [OR $1.495 \%$ confidence interval $(\mathrm{CI}) 0.7-2.8 ; P=0.30]$. Furthermore, up to one year after revascularization, no long-term benefit was observed in the late cardiac events. Although this study included a relatively small number of patients and was clearly underpowered for clinical events, it again suggests that, for improving postoperative outcomes in high-risk surgical patients, coronary revascularization does not offer a superior advantage to medical treatment.

In addition to the fact that revascularization did not protect patients against cardiac events in the CARP and DECREASE-V trials, PCI was associated with increased risk of MI compared with CABG in patients randomized to revascularization. ${ }^{61}$ Several other studies, with rates of death or MI over $20 \%$, have also raised concerns about deleterious, sometimes even catastrophic, effects of PCI, especially when stenting is performed prior to non-cardiac surgery. ${ }^{62-64}$ This is especially important for patients taking double antiplatelet therapy (ticlopidine or clopidogrel with aspirin), which cannot be maintained, ${ }^{62,63}$ or if the surgery is performed soon after stent implantation. ${ }^{64,65}$ However, if such antiplatelet therapy is maintained, the risk of bleeding may be increased following the surgery. ${ }^{64,66,67}$ Moreover, compared to patients without PAD, patients with PAD are at an increased risk of death and MI after PCI. ${ }^{68}$ Finally, CABG, which is associated with a 2 to $4 \%$ risk of perioperative death, ${ }^{46}$ did not improve long-term survival in the CARP trial. ${ }^{39}$ All of these findings suggest that there is no clear advantage in proceeding with revascularization prior to vascular surgery, if medical management is expected to yield similar protection.

\section{Management of antiplatelet therapy}

In patients with a previous PCI with stent implanta- tion, the management of antiplatelet therapy, in the setting of a non-cardiac surgery, represents a real challenge. In fact, premature discontinuation of a dual-antiplatelet therapy with aspirin and clopidogrel is associated with a non negligible risk of stent thrombosis. Several reports of drug-eluting stent (DES) thrombosis, occurring after cessation of antiplatelet therapy, have been documented, and are associated with a catastrophic case fatality rate of $20-45 \% .{ }^{69-71} \mathrm{~A}$ recent meta-analysis ${ }^{72}$ has demonstrated an incidence of early thrombosis (in the first 30 days) of 4.4/1,000 $(0.44 \%)$ with DES and of $5 / 1,000(0.5 \%)$ with baremetal stents (BMS) $(P=0.74)$. For late stent thrombosis (i.e., more than 30 days after implantation) the incidence was $5 / 1,000$ for DES vs 2.8/1,000 for BMS $(P=0.22)$. Median time of thrombosis occurrence was 15.5 months for sirolimus-eluting stent (173-773 days) and 18 months for paclitaxel-eluting stent (40-548 days). With a BMS, median thrombosis time was between 3.5 and four months. The risk of developing very late thrombosis phenomenon, (i.e., more than one year after implantation) appears to be restricted to DES, with an incidence of early thrombosis of $5 / 1,000(0.5 \%)$ vs $0 \%$ for BMS (relative risk $5.02, P=0.02) .{ }^{72}$ Clearly, these new findings influence the optimal duration of dual antiplatelet therapy.

Therefore, use of DES, in the context of an elective non-cardiac surgery, should be avoided. Consequently, the ACC/AHA guidelines recommend a combined antiplatelet treatment with aspirin and clopidogrel for a minimum of one month, for BMS, and for at least one year, for DES. ${ }^{35}$ It is also preferable to select a BMS in the setting of a planned surgery; however, only if it is clinically relevant, i.e., warranted independently of the perioperative context. In the case of an angioplasty performed with a balloon catheter alone, it is recommended to continue the perioperative administration of ASA and to delay surgery for two to four weeks, in order to allow healing of the injured vessel. ${ }^{73}$ The postponement of surgery by more than eight weeks could increase the risk of restenosis. ${ }^{35}$

A global evaluation must consider the hemorrhagic risk, if the antiplatelet therapy is continued, $v s$ the possible risk of a stent thrombosis, if the treatment is stopped. Therefore, it is important to facilitate a discussion between the surgeon, the anesthesiologist, and the cardiologist, in order to choose the better option for a given patient. It is also important to keep the patient informed of the suggested approach.

In the event that antiplatelet therapy needs to be prematurely discontinued after DES implantation, the risk of thrombosis will likely increase; therefore, it 
is strongly recommended to continue treatment with aspirin, at the very least. Taking into account platelet lifespan (seven days), a maximal interruption of five days for clopidogrel should be considered, in order to maintain some antiplatelet effect. Conversely, a complete interruption of at least five days is required, in order to avoid hemorrhagic complications (risk of major bleeding or major complication of even a minor bleeding). Obviously, double antiplatelet therapy should be resumed as soon as possible in the post-operative phase. ${ }^{35}$ The exact incidence of stent thrombosis in the perioperative remains unknown.

\section{Beta-blocker therapy}

Theoretically, $\beta$-blockers present several benefits. They enhance the reduction of myocardial $\mathrm{O}_{2}$ demand, prolong diastolic filling time, reduce arrhythmias, and provide protection against plaque rupture in the setting of activation of the sympathetic nervous system.

There is much controversy surrounding the perioperative use of $\beta$-blockers. No randomized study has specifically evaluated the importance of drug titration for a given heart rate or determined which $\beta$-blocker should be preferred. The optimal starting time and length of use also remain undetermined. In several randomized controlled trials $s^{74,75}$ and large observational studies, ${ }^{44,76}$ administration of $\beta$-blockers has been associated with important reductions in adverse clinical events and mortality following non-cardiac surgeries. However, more recent trials that focused on non-cardiac surgeries have failed to demonstrate the benefits of $\beta$-blockers. ${ }^{77-79}$ One of these published trials (MaVS) was larger, and focused on patients undergoing vascular surgery. Patients were treated during times starting at two hours, preoperatively, until a maximum of five days, postoperatively. The incidence of the primary endpoint, a composite of non-fatal MI, unstable angina, new congestive heart failure, new atrial or ventricular dysrythmia requiring treatment, or cardiac death at 30 days, did not differ significantly between the 246 patients who received metoprolol vs the 250 patients who received placebo (10.2\% vs $12.0 \%, P=0.57$ ). In a meta-analysis of 22 trials, including the MaVS trial results, Devereaux et al. ${ }^{80}$ found a non-significant, $56 \%$ reduction in cardiac events with $\beta$-blockers, and concluded that only a large randomized controlled trial would reveal the true value of $\beta$-blockers for patients undergoing highrisk surgery.

Very recently, the early results of the PeriOperative Ischemic Evaluation (POISE) trial were presented; they questioned the benefit and, even the safety, of preoperatively administered $\beta$-blockers. ${ }^{\text {B }}$ POISE was a randomized, double-blind clinical trial that included 4,174 patients in the metoprolol group (orally, 200 $\mathrm{mg} \cdot \mathrm{day}^{-1}$ of the sustained-released formula) and 4,177 patients in the placebo group. Previous CAD was found in $43 \%$ of patients, arterial vascular disease in $41 \%$ of patients, and history of stroke (cerebrovascular accident) in $15 \%$ of patients. Vascular surgery was the type of surgery performed in $42 \%$ of patients. Doses of metoprolol were not titrated, and the drug was stopped only if blood pressure dropped below 100 $\mathrm{mmHg}$. The incidence of primary outcome (combination of cardiovascular mortality, MI, or cardiac arrest) was lower in the metoprolol group vs the placebo group $[5.8 \%$ vs $6.9 \%$, hazard ratio (HR) $0.83 ; 95 \%$ CI, 0.70-0.99; $P=0.04$ ]. This positive outcome was primarily associated with a diminution in nonfatal infarcts $(3.6 \%$ vs $5.1 \%$; HR $0.70 ; P=0.0007)$. However, total mortality increased in the metoprolol group $(3.1 \%$ vs $2.3 \%$; HR $1.33 ; P=0.03)$, as were cerebrovascular accidents ( $1.0 \%$ vs $0.5 \%$; HR 2.17 ; $P=0.005)$. Patients in the metoprolol group also presented with more hypotension $(15.0 \%$ vs $9.7 \%$; $P<0.0001)$ and significant bradycardia (6.6\% vs $2.4 \%$; $P<0.0001)$. A major criticism of this study is that the dose of metoprolol might have been too high, with no consideration of blood pressure. In other words, $\beta$-blockers, if prescribed without consideration of blood pressure or $\mathrm{HR}$, might be harmful rather than protective.

Nevertheless, there remains indirect evidence to support the use of $\beta$-blockers, at least in patients undergoing vascular surgery. This evidence is derived from the two large randomized controlled trials that challenged the value of functional stratification (DECREASEII $)^{40}$ and coronary revascularization (CARP $)^{39}$ prior to vascular surgery vs medical treatment alone involving $\beta$-blocker therapy. In the CARP trial that failed to demonstrate benefits of revascularization prior to vascular surgery, $85 \%$ of patients were managed with $\beta$-blockers in the perioperative period. Use of statin therapy, angiotensin converting enzyme inhibitors, and ASA was also very high. Although it is not possible to attribute the absence of the clinical effect of revascularization solely to the fact that many patients received a $\beta$-blocker, we can at least conclude that good medical management, including the use of $\beta$-blockers, may alleviate the need for revascularization in the patients. ${ }^{39}$ Another source of indirect evidence supporting $\beta$ blocker therapy is the DECREASE-II study. ${ }^{40}$ All

B POISE Trial Results. Available from URL; http://www.medscape.com/viewarticle/565612. 
patients were managed with $\beta$-blockers, with a target heart rate between 60 and 65 beats. $\mathrm{min}^{-1}$ before surgery. The heart rate was below 50 beats. $\mathrm{min}^{-1}$ in $1.7 \%$ of the intermediate-risk patients and $>65$ beats $\cdot \mathrm{min}^{-1}$ in $16.5 \%$. Patients with a heart rate $<65$ beats. $\mathrm{min}^{-1}$ had a lower incidence of primary endpoints, i.e., composite of cardiac death and non-fatal MI 30 days postsurgery ( $1.3 \%$ vs $5.2 \%$ OR $0.24 ; 95 \%$ CI $0.09-0.66$; $P=0.003)$. Therefore, $\beta$-blockers do not work by "intention-to-treat"; a tightly controlled heart rate without hypotension seems to be a prerequisite for obtaining a valuable protective effect.

Despite the variability encountered in the results of these different studies, current ACC/AHA recommendations suggest the use of $\beta$-blockers in intermediate- to high-risk patients. This would include patients with $\geq$ two or three risk factors. ${ }^{35,81}$ However, it appears preferable to initiate treatment several days or weeks before surgery and to titrate the dose to reach a heart rate of $50-60$ beats $\mathrm{min}^{-1} \cdot{ }^{40}$ Cardioselective and prolonged action drugs may be more effective; therefore, atenolol or bisoprolol could be recommended. Indeed, the class effect of $\beta$-blockers in the perioperative setting ${ }^{82}$ and following $\mathrm{MI}^{83}$ has been challenged. In both studies, metoprolol was found to be inferior. Also, the large majority of the negative recent trials, including the very large POISE trial, used metoprolol. ${ }^{77-79, \mathrm{~B}}$ The optimal postoperative duration of treatment is unknown.

\section{Statin therapy}

Observational studies and one small randomized trial have suggested that statin therapy may be useful for the specific indication of perioperative protection against cardiac events. ${ }^{84-88}$ This may be related, in part, to anti-inflammatory properties, plaque stabilization effects, reduction of thrombogenesis, and improvement of endothelial function. The new ACC/AHA guidelines stipulate that statins should be continued in the perioperative period, if the patient is already on such therapy (class I). Statin use is reasonable for patients undergoing vascular surgery (class IIa). Finally, for patients with at least one clinical risk factor, who are undergoing intermediate-risk procedures, a statin therapy may be considered. (class IIb). ${ }^{35}$

\section{Acetylsalicylic acid (ASA) therapy}

One meta-analysis compared ASA to placebo in vascular surgery (infrainguinal bypass). A trend towards a reduction of events was observed. ${ }^{89}$ In contrast, the Pulmonary Embolism Prevention (PEP) trial with ASA in hip fracture patients suggested that there was an increased risk of cardiac ischemic events. ${ }^{90}$ The objective of the
PEP trial, however, was to demonstrate that ASA in orthopedic surgeries could prevent the occurrence of pulmonary embolism and deep venous thrombosis, not a reduction in cardiac events. Overall, very few cardiovascular events were observed, and, therefore, the statistical power to detect the impact of ASA on the occurrence of such events in PEP was limited.

On the other hand, for patients undergoing carotid endarterectomy, the latest American College of Chest Physicians (ACCP) guidelines recommended the initiation of ASA, preoperatively. ${ }^{91}$ In other types of vascular surgeries, the ideal timing to initiate ASA remains unclear. According to a recent review, ASA as monotherapy should not be routinely discontinued in cases of elective non-cardiac surgeries. ${ }^{92}$ For better graft patency, it is currently recommended to begin aspirin therapy prior to vascular reconstruction surgeries, although the protective effect is uncertain. ${ }^{91}$ For the time being, it is the clinician's task to assess whether the potential benefits outweigh the risk of bleeding secondary to ASA, when treatment is discontinued in the perioperative period.

\section{Conclusions and future directions}

In summary, identification and prevention of ischemia in patients undergoing major vascular surgery remains controversial. While the identification of preoperative ischemia is a marker of a higher perioperative risk, the value of identifying such ischemia has been questioned in intermediate-risk patients. However, appropriate management of patients, based on the results of such testing, is likely the key to improving their outcomes. Recent efforts aimed at reducing the ischemic consequences of severe coronary plaques (by revascularization or $\beta$-blockers therapy) have yielded conflicting results. Nevertheless, a diagnostic coronary angiography remains useful to assess adverse coronary anatomy such as LMD, when suspected based on functional testing. The role of non-invasive coronary angiography using MDCT, an emerging and promising technique, ${ }^{58}$ could help identify those high-risk patients. Additional studies will be needed in order to evaluate the role of MDCT in the non-cardiac perioperative setting. Alternative treatment strategies focusing on the overall atherosclerotic burden, including plaque stabilization and prevention of thrombosis, might result in improved clinical outcomes. In the non-surgical setting, plaque stabilization with statin therapy was associated with improved long-term outcomes compared to revascularization in one large trial. Observational studies and one randomized trial suggest a promising role for statin therapy in patients undergoing non-cardiac surgery, ${ }^{84-88}$ and larger clinical trials are underway. 
At this time, many questions remain unanswered regarding the impact of detecting inducible ischemia and the role of revascularization in patients managed with $\beta$-blockers undergoing vascular surgery. Consequently, further studies are needed to improve the perioperative risk stratification and management of patients undergoing vascular surgery.

\section{References}

1 Ashton CM, Petersen NJ, Wray NP, et al. The incidence of perioperative myocardial infarction in men undergoing noncardiac surgery. Ann Intern Med 1993; 118 : 504-10.

2 Lee TH, Marcantonio ER, Mangione CM, et al. Derivation and prospective validation of a simple index for prediction of cardiac risk of major noncardiac surgery. Circulation 1999; 100: 1043-9.

3 Devereaux PJ, Goldman L, Cook DJ, Gilbert K, Leslie $K$, Guyatt GH. Perioperative cardiac events in patients undergoing noncardiac surgery: a review of the magnitude of the problem, the pathophysiology of the events and methods to estimate and communicate risk. CMAJ 2005; 173: 627-34.

4 Krupski WC. Update on perioperative evaluation and management of cardiac disease in vascular surgery patients. J Vasc Surg 2002; 36: 1292-308.

5 Koskas F, Kieffer E. Long-term survival after elective repair of infrarenal abdominal aortic aneurysm: results of a prospective multicentric study. Association for Academic Research in Vascular Surgery (AURC). Ann Vasc Surg 1997; 11: 473-81.

6 Fleisher LA, Eagle KA, Shaffer T, Anderson GF. Perioperative- and long-term mortality rates after major vascular surgery: the relationship to preoperative testing in the medicare population. Anesth Analg 1999; 89: 849-55.

7 Shah KB, Kleinman BS, Rao TL, Jacobs HK, Mestan K, Schaafsma $M$. Angina and other risk factors in patients with cardiac diseases undergoing noncardiac operations. Anesth Analg 1990; 70: 240-7.

8 Mangano DT, Browner WS, Hollenberg M, Li J, Tateo $I M$. Long-term cardiac prognosis following noncardiac surgery. The Study of Perioperative Ischemia Research Group. JAMA 1992; 268: 233-9.

9 Alpert JS, Thygesen K, Antman E, Bassand JP. Myocardial infarction redefined--a consensus document of The Joint European Society of Cardiology/American College of Cardiology Committee for the redefinition of myocardial infarction. J Am Coll Cardiol 2000; 36: 959-69.

10 Landesberg G, Shatz V, Akopnik I, et al. Association of cardiac troponin, CK-MB, and postoperative myocardial ischemia with long-term survival after major vascular surgery. J Am Coll Cardiol 2003; 42: 1547-54.
11 Liu LL, Wiener-Kronish JP. Preoperative cardiac evaluation of women for noncardiac surgery. Cardiol Clin 1998; 16: 59-66.

12 Eagle KA, Berger PB, Calkins $H$, et al. ACC/AHA guideline update for perioperative cardiovascular evaluation for noncardiac surgery--executive summary a report of the American College of Cardiology/American Heart Association Task Force on Practice Guidelines (Committee to Update the 1996 Guidelines on Perioperative Cardiovascular Evaluation for Noncardiac Surgery). Circulation 2002; 105: 1257-67.

13 Devereaux PJ, Goldman L, Yusuf S, Gilbert K, Leslie K, Guyatt GH. Surveillance and prevention of major perioperative ischemic cardiac events in patients undergoing noncardiac surgery: a review. CMAJ 2005; 173: 779-88.

14 Falk E. Plaque rupture with severe pre-existing stenosis precipitating coronary thrombosis. Characteristics of coronary atherosclerotic plaques underlying fatal occlusive thrombi. Br Heart J 1983; 50: 127-34.

15 Horie T, Sekiguchi M, Hirosawa K. Coronary thrombosis in pathogenesis of acute myocardial infarction. Histopathological study of coronary arteries in 108 necropsied cases using serial section. Br Heart J 1978; 40: 153-61.

16 Falk E, Shah PK, Fuster V. Coronary plaque disruption. Circulation 1995; 92: 657-71.

17 Little WC, Constantinescu M, Applegate RJ, et al. Can coronary angiography predict the site of a subsequent myocardial infarction in patients with mild-to-moderate coronary artery disease? Circulation 1988; 78: 1157-66.

18 Giroud D, Li JM, Urban P, Meier B, Rutishauer W. Relation of the site of acute myocardial infarction to the most severe coronary arterial stenosis at prior angiography. Am J Cardiol 1992; 69: 729-32.

19 Mintz GS, Painter JA, Pichard AD, et al. Atherosclerosis in angiographically "normal" coronary artery reference segments: an intravascular ultrasound study with clinical correlations. J Am Coll Cardiol 1995; 25: 1479-85.

20 Alderman EL, Corley SD, Fisher LD, et al. Five-year angiographic follow-up of factors associated with progression of coronary artery disease in the Coronary Artery Surgery Study (CASS). CASS Participating Investigators and Staff. J Am Coll Cardiol 1993; 22: 1141-54.

21 Davies MJ, Bland JM, Hangartner JR, Angelini A, Thomas $A C$. Factors influencing the presence or absence of acute coronary artery thrombi in sudden ischaemic death. Eur Heart J 1989; 10: 203-8.

22 Mann JM, Davies MJ. Vulnerable plaque. Relation of characteristics to degree of stenosis in human coronary arteries. Circulation 1996; 94: 928-31.

23 Ge J, Chirillo F, Schwedtmann J, et al. Screening of ruptured plaques in patients with coronary artery disease by intravascular ultrasound. Heart 1999; 81: 621-7. 
24 von Birgelen $C$, Klinkhart W, Mintz GS, et al. Plaque distribution and vascular remodeling of ruptured and nonruptured coronary plaques in the same vessel: an intravascular ultrasound study in vivo. J Am Coll Cardiol 2001; 37: 1864-70.

25 Ellis SG, Hertzer NR, Young JR, Brener S. Angiographic correlates of cardiac death and myocardial infarction complicating major nonthoracic vascular surgery. Am J Cardiol 1996; 77: 1126-8.

26 Dawood MM, Gutpa DK, Southern J, Walia A, Atkinson $J B$, Eagle $K A$. Pathology of fatal perioperative myocardial infarction: implications regarding pathophysiology and prevention. Int J Cardiol 1996; 57: 37-44.

27 Cohen MC, Aretz TH. Histological analysis of coronary artery lesions in fatal postoperative myocardial infarction. Cardiovasc Pathol 1999; 8: 133-9.

28 Landesberg G, Mosseri M, Zahger D, et al. Myocardial infarction after vascular surgery: the role of prolonged stress-induced, ST depression-type ischemia. J Am Coll Cardiol 2001; 37: 1839-45.

29 Davenport DL, Ferraris VA, Hosokawa P, Henderson $W G$, Khuri SF, Mentzer RM Jr. Multivariable predictors of postoperative cardiac adverse events after general and vascular surgery: results from the patient safety in surgery study. J Am Coll Surg 2007; 204: 1199-210.

30 Rinfret S, Goldman L, Polanczyk CA, Cook EF, Lee TH. Value of immediate postoperative electrocardiogram to update risk stratification after major noncardiac surgery. Am J Cardiol 2004; 94: 1017-22.

31 Kertai MD, Boersma E, Klein J, Van Urk H, Bax JJ, Poldermans D. Long-term prognostic value of asymptomatic cardiac troponin $\mathrm{T}$ elevations in patients after major vascular surgery. Eur J Vasc Endovasc Surg 2004; 28: 59-66.

32 Bursi F, Babuin L, Barbieri A, et al. Vascular surgery patients: perioperative and long-term risk according to the ACC/AHA guidelines, the additive role of post-operative troponin elevation. Eur Heart J 2005; 26: 2448-56.

33 Kim LJ, Martinez EA, Faraday N, et al. Cardiac troponin I predicts short-term mortality in vascular surgery patients. Circulation 2002; 106: 2366-71.

34 Higham H, Sear JW, Sear YM, Kemp M, Hooper RJ, Foex $P$. Peri-operative troponin I concentration as a marker of long-term postoperative adverse cardiac outcomes - a study in high-risk surgical patients. Anaesthesia 2004; 59: 318-23.

35 Fleisher LA, Beckman JA, Brown KA, et al. ACC/ AHA 2007 guidelines on perioperative cardiovascular evaluation and care for noncardiac surgery: a report of the American College of Cardiology/American Heart Association Task Force on Practice Guidelines (Writing Committee to Revise the 2002 Guidelines on Perioperative Cardiovascular Evaluation for Noncardiac Surgery) developed in collaboration with the American Society of Echocardiography, American Society of Nuclear Cardiology, Heart Rhythm Society, Society of Cardiovascular Anesthesiologists, Society for Cardiovascular Angiography and Interventions, Society for Vascular Medicine and Biology, and Society for Vascular Surgery. J Am Coll Cardiol 2007; 50: el59-241.

36 Eagle KA, Coley CM, Newell JB, et al. Combining clinical and thallium data optimizes preoperative assessment of cardiac risk before major vascular surgery. Ann Intern Med 1989; 110: 859-66.

37 Lette J, Waters D, Lassonde J, et al. Multivariate clinical models and quantitative dipyridamole-thallium imaging to predict cardiac morbidity and death after vascular reconstruction. J Vasc Surg 1991; 14: 160-9.

38 Rao TL, Jacobs KH, El-Etr AA. Reinfarction following anesthesia in patients with myocardial infarction. Anesthesiology 1983; 59: 499-505.

39 McFalls EO, Ward HB, Moritz TE, et al. Coronaryartery revascularization before elective major vascular surgery. N Engl J Med 2004; 351: 2795-804.

40 Poldermans D, Bax JJ, Schouten O, et al. Should major vascular surgery be delayed because of preoperative cardiac testing in intermediate-risk patients receiving beta-blocker therapy with tight heart rate control? J Am Coll Cardiol 2006; 48: 964-9.

41 Coley CM, Field TS, Abraham SA, Boucher CA, Eagle $K A$. Usefulness of dipyridamole-thallium scanning for preoperative evaluation of cardiac risk for nonvascular surgery. Am J Cardiol 1992; 69: 1280-5.

42 Underwood SR, Anagnostopoulos C, Cerqueira M, et al. Myocardial perfusion scintigraphy: the evidence. Eur J Nucl Med Mol Imaging 2004; 31: 261-91.

43 Kertai MD, Boersma E, Bax JJ, et al. A meta-analysis comparing the prognostic accuracy of six diagnostic tests for predicting perioperative cardiac risk in patients undergoing major vascular surgery. Heart 2003; 89: 1327-34.

44 Boersma E, Poldermans D, Bax JJ, et al. Predictors of cardiac events after major vascular surgery: role of clinical characteristics, dobutamine echocardiography, and beta-blocker therapy. JAMA 2001; 285: 1865-73.

45 Etchells E, Meade M, Tomlinson G, Cook D. Semiquantitative dipyridamole myocardial stress perfusion imaging for cardiac risk assessment before noncardiac vascular surgery: a meta-analysis. J Vasc Surg 2002; 36: 534-40.

46 Eagle KA, Guyton RA, Davidoff R, et al. ACC/AHA 2004 guideline update for coronary artery bypass graft surgery: a report of the American College of Cardiology/American Heart Association Task Force on Practice Guidelines (Committee to Update the 1999 Guidelines for Coronary Artery Bypass Graft Surgery). Circulation 2004; 110: e340-437.

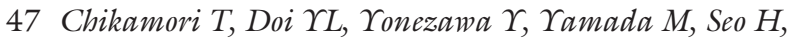


Ozawa $T$. Noninvasive identification of significant narrowing of the left main coronary artery by dipyridamole thallium scintigraphy. Am J Cardiol 1991; 68: 472-7.

48 Nygaard TW, Gibson RS, Ryan JM, Gascho JA, Watson $D D$, Beller GA. Prevalence of high-risk thallium-201 scintigraphic findings in left main coronary artery stenosis: comparison with patients with multiple- and singlevessel coronary artery disease. Am J Cardiol 1984; 53 : 462-9.

49 Rehn T, Griffith LS, Achuff SC, et al. Exercise thallium201 myocardial imaging in left main coronary artery disease: sensitive but not specific. Am J Cardiol 1981; 48: 217-23.

50 Kostkiewicz M, Tracz W, Przewlocki T, Kawalec E. Predictive potential of noninvasive methods, inclusive of exercise SPECT Tc99m MIBI imaging, in recognition of high-risk patients with left main coronary artery stenosis. Int J Cardiovasc Imaging 2001; 17: 347-52.

51 Wakasugi S, Shibata N, Kobayashi T, Fudemoto $\Upsilon$, Hasegawa $\Upsilon$, Nakano S. Specific perfusion pattern in stress $201 \mathrm{Tl}$ myocardial scintigraphy of left main coronary artery disease. Eur J Nucl Med 1986; 12: 369-74.

52 Mahajan NM, Thekkott DT, Hollander GH, Greengart AG, Shani JS, Lichstein EL. Role of nuclear stress test as a noninvasive tool in detection of isolated and significant left main coronary artery disease : there is no unique pattern of perfusion deficit. J Nuclear Cardiol 2005; 12: s121.

53 Andrade MJ, Picano E, Pingitore A, et al. Dipyridamole stress echocardiography in patients with severe left main coronary artery narrowing. Echo Persantine International Cooperative (EPIC) Study Group--Subproject «Left Main Detection». Am J Cardiol 1994; 73: 450-5.

54 Thompson C, Bergstrome D, Parlow JL. Limitations of preoperative dobutamine stress echocardiography in identifying severe left main coronary artery stenosis: a report of two cases and a brief review. Can J Anesth 2003; 50: 933-9.

55 Pierpont GL, Moritz TE, Goldman S, et al. Disparate opinions regarding indications for coronary artery revascularization before elective vascular surgery. Am J Cardiol 2004; 94: 1124-8.

56 Boden WE, O'Rourke RA, Teo KK, et al. Optimal medical therapy with or without PCI for stable coronary disease. N Engl J Med 2007; 356: 1503-16.

57 Hertzer NR, Beven EG, Young JR, et al. Coronary artery disease in peripheral vascular patients. A classification of 1000 coronary angiograms and results of surgical management. Ann Surg 1984; 199: 223-33.

58 Budoff MJ, Achenbach S, Blumenthal RS, et al. Assessment of coronary artery disease by cardiac computed tomography: a scientific statement from the Amer- ican Heart Association Committee on Cardiovascular Imaging and Intervention, Council on Cardiovascular Radiology and Intervention, and Committee on Cardiac Imaging, Council on Clinical Cardiology. Circulation 2006; 114: 1761-91.

59 Foster ED, Davis KB, Carpenter JA, Abele S, Fray D. Risk of noncardiac operation in patients with defined coronary disease: The Coronary Artery Surgery Study (CASS) registry experience. Ann Thorac Surg 1986; 41: 42-50.

60 Hassan SA, Hlatky MA, Boothroyd DB, et al. Outcomes of noncardiac surgery after coronary bypass surgery or coronary angioplasty in the Bypass Angioplasty Revascularization Investigation (BARI). Am J Med 2001; 110: 260-6.

61 Ward HB, Kelly RF, Thottapurathu L, et al. Coronary artery bypass grafting is superior to percutaneous coronary intervention in prevention of perioperative myocardial infarctions during subsequent vascular surgery. Ann Thorac Surg 2006; 82: 795-800; discussion 800-1.

62 Kaluza GL, Joseph J, Lee JR, Raizner ME, Raizner AE. Catastrophic outcomes of noncardiac surgery soon after coronary stenting. J Am Coll Cardiol 2000; 35: 1288-94.

63 Schouten O, van Domburg RT, Bax JJ, et al. Noncardiac surgery after coronary stenting: early surgery and interruption of antiplatelet therapy are associated with an increase in major adverse cardiac events. J Am Coll Cardiol 2007; 49: 122-4.

64 Vicenzi MN, Meislitzer T, Heitzinger B, Halaj M, Fleisher LA, Metzler $H$. Coronary artery stenting and non-cardiac surgery--a prospective outcome study. Br J Anaesth 2006; 96: 686-93.

65 Wilson SH, Fasseas P, Orford JL, et al. Clinical outcome of patients undergoing non-cardiac surgery in the two months following coronary stenting. J Am Coll Cardiol 2003; 42: 234-40.

66 Chapman TW, Bowley DM, Lambert AW, Walker AJ, Ashley SA, Wilkins DC. Haemorrhage associated with combined clopidogrel and aspirin therapy. Eur J Vasc Endovasc Surg 2001; 22: 478-9.

67 Dalal AR, D'Souza S, Shulman MS. Brief review: Coronary drug-eluting stents and anesthesia. Can J Anesth 2006; 53: 1230-43.

68 Saw J, Bhatt DL, Moliterno DJ, et al. The influence of peripheral arterial disease on outcomes: a pooled analysis of mortality in eight large randomized percutaneous coronary intervention trials. J Am Coll Cardiol 2006; 48: 1567-72.

69 Spertus JA, Kettelkamp R, Vance C, et al. Prevalence, predictors, and outcomes of premature discontinuation of thienopyridine therapy after drug-eluting stent place- 
ment: results from the PREMIER registry.

Circulation 2006; 113: 2803-9.

70 Pfisterer M, Brunner-La Rocca HP, Buser PT, et al. Late clinical events after clopidogrel discontinuation may limit the benefit of drug-eluting stents: an observational study of drug-eluting versus bare-metal stents. J Am Coll Cardiol 2006; 48: 2584-91.

71 Eisenstein EL, Anstrom KJ, Kong DF, et al. Clopidogrel use and long-term clinical outcomes after drug-eluting stent implantation. JAMA 2007; 297: 159-68.

72 Bavry AA, Kumbhani DJ, Helton TJ, Borek PP, Mood $G R$, Bhatt DL. Late thrombosis of drug-eluting stents: a meta-analysis of randomized clinical trials. Am J Med 2006; 119: 1056-61.

73 Brilakis ES, Orford JL, Fasseas P, et al. Outcome of patients undergoing balloon angioplasty in the two months prior to noncardiac surgery. Am J Cardiol 2005; 96: 512-4.

74 Poldermans D, Boersma E, Bax JJ, et al. The effect of bisoprolol on perioperative mortality and myocardial infarction in high-risk patients undergoing vascular surgery. Dutch Echocardiographic Cardiac Risk Evaluation Applying Stress Echocardiography Study Group. N Engl J Med 1999; 341: 1789-94.

75 Mangano DT, Layug EL, Wallace A, Tateo I. Effect of atenolol on mortality and cardiovascular morbidity after noncardiac surgery. Multicenter Study of Perioperative Ischemia Research Group. N Engl J Med 1996; 335: 1713-20.

76 Lindenawer PK, Pekow P, Wang K, Mamidi DK, Gutierrez B, Benjamin EM. Perioperative beta-blocker therapy and mortality after major noncardiac surgery. N Engl J Med 2005; 353: 349-61.

77 Brady AR, Gibbs JS, Greenhalgh RM, Powell JT, Sydes MR; POBBLE Trial Investigators. Perioperative betablockade (POBBLE) for patients undergoing infrarenal vascular surgery: results of a randomized double-blind controlled trial. J Vasc Surg 2005; 41: 602-9.

78 Junl AB, Wetterslev J, Glund C, et al. Effect of perioperative beta blockade in patients with diabetes undergoing major non-cardiac surgery: randomised placebo controlled, blinded multicentre trial. BMJ 2006; 332: 1482.

79 Yang H, Raymer K, Butler R, Parlow J, Roberts $R$. The effects of perioperative beta-blockade: results of the Metoprolol after Vascular Surgery (MaVS) study, a randomized controlled trial. Am Heart J 2006; 152: 983-90.

80 Devereaux PJ, Beattie WS, Choi PT, et al. How strong is the evidence for the use of perioperative beta blockers in non-cardiac surgery? Systematic review and meta-analysis of randomised controlled trials. BMJ 2005; 331: 313-21.
81 Auerbach A, Goldman L. Assessing and reducing the cardiac risk of noncardiac surgery. Circulation 2006; 113: 1361-76.

82 Redelmeier D, Scales D, Kopp A. Beta blockers for elective surgery in elderly patients: population based, retrospective cohort study. BMJ 2005; 331: 932.

83 Rinfret $S$, Abrahamowicz M, Tu J, et al. A populationbased analysis of the class effect of beta-blockers after myocardial infarction. Am Heart J 2007; 153: 224-30.

84 Clark LL, Ikonomidis JS, Crawford FA Jr, et al. Preoperative statin treatment is associated with reduced postoperative mortality and morbidity in patients undergoing cardiac surgery: an 8-year retrospective cohort study. J Thorac Cardiovasc Surg 2006; 131: 679-85.

85 Lindenauer PK, Pekow P, Wang K, Gutierrez B, Benjamin EM. Lipid-lowering therapy and in-hospital mortality following major noncardiac surgery. JAMA 2004; 291: 2092-9.

86 O'Neil-Callahan K, Katsimaglis G, Tepper MR, et al. Statins decrease perioperative cardiac complications in patients undergoing noncardiac vascular surgery: the Statins for Risk Reduction in Surgery (StaRRS) study. J Am Coll Cardiol 2005; 45: 336-42.

87 Hindler K, Eltzschig HK, Fox AA, Body SC, Shernan $S K$, Collard CD. Influence of statins on perioperative outcomes. J Cardiothorac Vasc Anesth 2006; 20: 251-8

88 Durazzo AE, Machado FS, Ikeoka DT, et al. Reduction in cardiovascular events after vascular surgery with atorvastatin: a randomized trial. J Vasc Surg 2004; 39 : 967-75; discussion 975-6.

89 Robless P, Mikhailidis DP, Stansby G. Systematic review of antiplatelet therapy for the prevention of myocardial infarction, stroke or vascular death in patients with peripheral vascular disease. Br J Surg 2001; 88: 787-800.

90 Anonymous. Prevention of pulmonary embolism and deep vein thrombosis with low dose aspirin: Pulmonary Embolism Prevention (PEP) trial. Lancet 2000; 355: 1295-302.

91 Clagett GP, Sobel M, Jackson MR, Lip GY, Tangelder M, Verhaeghe R. Antithrombotic therapy in peripheral arterial occlusive disease: the Seventh ACCP Conference on Antithrombotic and Thrombolytic Therapy. Chest 2004; 126: 609S-26S.

92 Merritt JC, Bhatt DL. The efficacy and safety of perioperative antiplatelet therapy. J Thromb Thrombolysis 2004; 17: 21-7. 